\title{
Estimating the Spectrum of Leak Noise in Buried Plastic Water Distribution Pipes using Acoustic or Vibration Measurements Remote from the Leak
}

\author{
O. Scussel $^{a *}$, M.J. Brennan ${ }^{a}$, F.C.L Almeida ${ }^{b, c}$ J.M. Muggleton $^{d}$, \\ E. Rustighi ${ }^{d}$, P.F. Joseph ${ }^{d}$
}

\footnotetext{
${ }^{a}$ Department of Mechanical Engineering, UNESP-FEIS, Ilha Solteira, São Paulo, 15385000, Brazil.

b Faculty of Science and Engineering, UNESP-FCE, Tupã, São Paulo, 17602-496, Brazil.

c Department of Mechanical Engineering, UNESP-FEB, Bauru, São Paulo, 17033-360, Brazil.

${ }^{\mathrm{d}}$ Institute of Sound and Vibration Research, University of Southampton, Highfield, SO17 1BJ, Southampton, United Kingdom.

* Corresponding author email: oscar.scussel@gmail.com
}

No of pages: 21

No of figures: 6

No of tables: 3

\section{Highlights}

- A method to estimate the spectrum of leak noise at source is presented.

- The proposed method is applied to data from three test sites.

- The effects of using hydrophones or accelerometers on the estimation of the leak noise spectrum are highlighted. 


\begin{abstract}
An appropriate model of leak noise at source is necessary in analytical and numerical approaches to investigate the characteristics of leak noise measured remotely from the leak in buried water pipes. It is extremely difficult to measure leak noise at source in practice, so an inverse method is needed to predict this from measurements made either side of the leak at convenient access points. This paper presents such a method, and illustrates the approach using four data sets from three different test sites. The method requires that the noise propagates in the pipe according to a simple model of wave propagation within the frequency range over which leak noise is detected at the sensors. Using the measured data, the real and imaginary parts of the wavenumber are estimated, and these, together with an estimated position of the leak between the two sensors, the frequency response functions corresponding to the sections of the pipe either side of the leak position are estimated. If pressure measurements are made, then both the level and shape of the leak noise spectrum can be estimated, but if accelerometers are used then only an estimate of the shape of the spectrum is possible. From the measurements presented, it is found that it is not possible to state categorically that the leak noise spectra decays according to a particular frequency power law. There is some evidence that it decays with a frequency power law of $\omega^{-1}$, which agrees with previous laboratory based experiments, but this is not definitive in all cases.
\end{abstract}




\section{Introduction}

In recent years, water scarcity has become a topic of great concern due to a steadily increasing demand for clean drinking water and decrease in water supplies, resulting in potential social, environmental and economic effects [1]. It has been estimated that in many water distribution networks around the world, the loss of pumped water can frequently exceed $30 \%$ of the input volume. In some cases it reaches much higher levels, from $40 \%$ to $50 \%$ due to leaks or pipe breaks resulting from holes, deterioration and damage [2-4].

To ameliorate some of the problems, advances in leak detection/location methods have occurred in recent years, a review of which is given in [2]. Among the existing techniques to locate leaks, vibro-acoustic methods have proved to be useful, generally giving satisfactory results, though for plastic pipes, these techniques have been less effective than with metal pipes due to much higher attenuation of leak noise propagating to the measurement points $[5,6]$. To determine the mechanisms of vibro-acoustic energy loss in plastic pipes, analytical and numerical models have been developed, with some degree of success, for example $[7,8]$. Cross-correlation methods have been successful in location leaks, for example [6,9], Alternative methods using support vector machines and probabilistic models have also been used [10], together with predictive machine learning algorithms to predict leakage flow rate in plastic water pipes [11]. Another data-driven anomaly detection approach has been investigated by Cody et al. [12] to extract leaksensitive features for leak detection and location.

In models that involve leak noise propagation and measurement, the spectral characteristics of the leak noise at source have to be assumed. Frequently, it is assumed 
that the leak spectrum can be represented by white noise, but there is very little experimental data to support this assumption. Some work has been carried out on leak noise characterisation in in-air rather than buried pipes [11,13,14]. Butterfield et al. $[11,13]$ showed that the leak flow rate as well as the size of the leak has an influence on the spectrum of the measured leak noise. Moreover, they found that hydrophones provide a more accurate predictions for plastic pipes, compared with accelerometers. Papastefanou et al. [14], presented an experimental investigation into the characteristics of the leak noise spectrum at source, and how it is affected by the leak flow velocity and the size of the leak. Leak noise measurements using hydrophones were made, and an empirical model for the leak noise spectrum was proposed. They found that the leak spectrum within the pipe generally decays with frequency $\omega$ at a rate of $\omega^{-1}$ up to some critical frequency related to the exit flow velocity and leak size, above which it decays at the much faster rate of $\omega^{-5}$.

The fundamental problem with trying to estimate the spectral characteristics of leak noise at source in a buried pipe is the practical difficulty of placing a sensor inside the pipe at the leak location. In this paper an alternative approach is proposed that uses measurements made either side of the leak at convenient access points. This approach is similar to that described in [14], but there are important differences in the way in which the pipe-system is modelled, and it is applied to three buried pipe systems in different countries rather than an in-air pipe in the laboratory.

The aim of the paper is to present a method to estimate the leak noise spectrum at source from measured data, typically made using leak noise correlators. As these involve different types of sensors, for example hydrophones or accelerometers, the approach 
considers the sensor type. The method proposed can predict the spectral shape within a frequency bandwidth where the leak noise propagates as a nondispersive wave. The paper focusses on buried plastic water pipes. With such pipes leak noise measured using hydrophones has been found to be highly correlated to accelerometer measurements of the pipe-wall or pipe fittings $[15,16]$. The method proposed can therefore predict the spectral shape of the leak noise at source for both types of sensors. Additionally, when hydrophones are used, the amplitude of the spectrum can be estimated. Four measurements, from three different test sites, are used to illustrate the approach.

The paper is organized as follows. Following this introduction, Section 2 describes the method by which the leak noise spectrum can be estimated from measurements made either side of the leak. Sections 3 and 4 describe the experimental test-rigs and the results respectively. A discussion of the results and the limitations of the method are given in Section 5 and some conclusions are given in Section 6.

\section{Estimation of the Leak Noise Spectrum}

Fig. 1 shows a typical situation in which an acoustic correlator is used to locate the position of a suspected water leak. In such a situation, vibration (accelerometers, geophones) or acoustic (hydrophones) sensors are attached to convenient access points (measurement positions), such as hydrants or valves, either side of the suspected leak position, to measure two signals $x_{1}(t)$ and $x_{2}(t)$, which are the responses of the pipe system at the measurement positions, due to the leak noise. These signals are filtered versions of the leak noise, which has propagated through the pipe to reach the sensors, both of which can have a profound effect on the measured noise. As the signals $x_{1}(t)$ and $x_{2}(t)$ are readily available when using leak noise correlators, the question addressed in 
this paper is whether these signals, together with a model of the pipe system and sensors, can be used to estimate the noise spectrum at the leak position?

The pipe measurement system shown in Fig.1(a) is essentially a single input, two output system as shown in block diagram form in Fig. 1(b), where $l(t)$ is the time history of the leak noise at source. Here $l(t)$ is taken to be the equivalent pressure time history that is able to propagate many acoustic wavelengths from the leak. In practice, a measurement of the leak signal directly at the leak position may contain contributions to the pressure from the non-radiating near field of the leak.

The terms $H_{1}\left(\omega, d_{1}\right)$ and $H_{2}\left(\omega, d_{2}\right)$ are the Frequency Response Functions (FRFs) between the acoustic pressure of the leak noise and the acoustic pressure at the measurement positions either side of the leak. It is assumed that the same type of sensor is used at each measurement position, and the FRF of this sensor is given by $H_{s}(\omega)$. If hydrophones are used as sensors then $H_{s}(\omega)=1$, and if accelerometers are used as sensors then $H_{s}(\omega)=\alpha \omega^{2}$, where $\alpha$ is a constant [15]. This is because the radial displacement of the pipe is proportional to the internal acoustic pressure. In practice the radial displacement of the pipe is not measured because it is not readily accessible, but the acceleration of the pipe fitting which is measured is found to be strongly coupled to this in practice.

The Power Spectral Density (PSD) of the leak noise at source $S_{l l}(\omega)$, is related to Cross Power Spectral Density (CPSD) $S_{x_{1} x_{2}}(\omega)$, between the measured signals and the estimated FRFs by [6] 


$$
S_{l l}(\omega)=\frac{1}{\left|H_{s}(\omega)\right|^{2}} \frac{S_{x_{1} x_{2}}(\omega)}{H_{1}^{*}\left(\omega, d_{1}\right) H_{2}\left(\omega, d_{2}\right)}
$$

where * denotes the complex conjugate, or by the PSDs $S_{x_{1} x_{1}}(\omega)$ or $S_{x_{2} x_{2}}(\omega)$ of the measured signals by

$$
S_{l l}(\omega)=\frac{S_{x_{1} x_{1}}(\omega)}{\left|H_{s}(\omega) H_{1}\left(\omega, d_{1}\right)\right|^{2}} \quad \text { and } \quad S_{l l}(\omega)=\frac{S_{x_{2} x_{2}}(\omega)}{\left|H_{s}(\omega) H_{2}\left(\omega, d_{2}\right)\right|^{2}}
$$

Although it is possible to use any of the Eqs. $(1 a, b, c)$ to estimate the spectrum of the leak noise, it is preferable to use Eq. (1a) as it uses the CPSD between the measurements signals $x_{1}(t)$ and $x_{2}(t)$, rather than their PSDs, so uncorrelated noise between the two measurements is filtered out.

To estimate the FRFs $H_{1}\left(\omega, d_{1}\right)$ and $H_{2}\left(\omega, d_{2}\right)$, the distances $d_{1}$ and $d_{2}$ need to be estimated first. This can be done by using the technique employed in leak noise correlators, which estimates the difference in the arrival times (time delay) $T_{0}$, of the leak noise at the measurement positions. Hence, using $T_{0}$, together with the speed of the leak noise propagation $c$ along the pipe, and the distance between the measurement positions $d$, the position of the leak can be determined by [6]:

$$
d_{2}=\frac{d-c T_{0}}{2}
$$


Note, that leak noise in plastic pipes generally propagates as a predominantly fluid-borne wave, which is strongly coupled to the radial motion of the pipe-wall [7,8]. In Eq. (2), the speed of leak noise propagation is commonly determined from tables, and the time delay is determined from the peak in the Cross-Correlation Function (CCF), which is given by [6]

$$
R_{x_{1} x_{2}}(\tau)=F^{-1}\left\{S_{x_{1} x_{2}}(\omega)\right\}=\frac{1}{2 \pi} \int_{-\infty}^{+\infty} S_{x_{1} x_{2}}(\omega) e^{\mathrm{j} \omega \tau} d \omega
$$

where $\mathrm{j}=\sqrt{-1}$ and $F^{-1}\{\bullet\}$ denotes the inverse Fourier transform. To estimate the FRFs, it is assumed that a wave propagates from the source of the leak to each measurement position without reflection, so that

$$
H_{1}\left(\omega, d_{1}\right)=e^{\mathrm{j} k d_{1}} \quad \text { and } \quad H_{2}\left(\omega, d_{2}\right)=e^{\mathrm{j} k d_{2}}
$$

where $k$ is the complex wavenumber, corresponding to the predominantly fluid-borne wave that propagates the leak noise. The real and the imaginary parts of the wavenumber are estimated from the FRF between the measured signals $x_{1}(t)$ and $x_{2}(t)$, which is given by

$$
H_{x_{1} X_{2}}(\omega)=\frac{S_{x_{1} X_{2}}(\omega)}{S_{X_{1} X_{1}}(\omega)}
$$

The real part of the wavenumber $\operatorname{Re}\{k\}$ is estimated from the phase of $H_{x_{1} x_{2}}(\omega)$, and is given by [8] 


$$
\operatorname{Re}\{k\}=\frac{\arg \left(H_{x_{1} x_{2}}(\omega)\right)}{d_{1}-d_{2}}
$$

The imaginary part of the wavenumber is estimated from the modulus of $H_{x_{1} x_{2}}(\omega)$ and is given by [8]

$$
\operatorname{Im}\{k\}=\frac{\ln \left|H_{x_{1} x_{2}}(\omega)\right|}{d_{1}-d_{2}}
$$

Once the distances $d_{1}$ and $d_{2}$, and the real and imaginary parts of the wavenumber have been estimated, the FRFs given in Eqs. $(4 a, b)$ can be estimated. Provided that the physical system behaves in accordance with the assumed model, such that the propagation of the leak noise can be accurately represented by transfer-functions, then the spectral shape of the leak can be determined using Eqs. (1a, b or c), with a preference for Eq. (1a) as discussed previously. If calibrated hydrophones are used as sensors then the magnitude of the leak noise spectrum can be estimated. However, if accelerometers are used then an estimate of the magnitude of the leak noise spectrum is not possible as the constant $\alpha$ is not known (Note that it is assumed in this paper that this constant is the same at each sensor position). Only the shape of the spectrum can be estimated in this case.

\section{Experimental work}

In this Section, the method of estimating the leak noise spectrum is applied to data sets obtained from three different pipe systems (test sites) under very different conditions. 
Tabs. 1 and 2 give the pipe and soil properties of the three test sites. Descriptions of each experimental test rig and details of data collection methods are given in this section.

The test rigs are located in Ottawa (Canada), Blithfield (UK) and São Paulo (Brazil), which have different soil properties, together with the pipe geometry and material characteristics according to their locations. Their schematic diagrams are shown in Figs. 2(a,b,c) respectively. Fig. 2 also depicts the way in which the leak was simulated and the positions at which the leak noise measurements were made for each test rig. In each case, measurements were carried out using accelerometers, but hydrophones were also used in the Canadian system. The photographs in Fig. 2 show the leak mechanisms together with the sensor arrangements. Moreover, the photographs of the leaks in the Ottawa and São Paulo test rigs, show the pipes before they were buried.

\section{Ottawa test rig}

Several studies have been conducted using the data from the Ottawa test rig, and more details on this test rig can be found in $[5,6,17]$. The test section of the polyvinyl chloride (PVC) pipe is approximately $200 \mathrm{~m}$ long and is buried at depth of $2.4 \mathrm{~m}$. It has an outer radius and wall thickness of $75 \mathrm{~mm}$ and $9.85 \mathrm{~mm}$, respectively. The measurement positions were at access points $\mathrm{P} 1$ and P2, which are $102.6 \mathrm{~m}$ apart, with the leak located $29.1 \mathrm{~m}$ from P1, as shown in Fig. 2(a). The access points are above ground and are connected to the buried pipe by risers. The leak was generated by way of a crack in the pipe and the vibration was measured using accelerometers and hydrophones placed on hydrants, as shown in Fig. 2(a). Two 66 second synchronised time histories were measured at access points P1 and P2 using a sampling frequency of $500 \mathrm{~Hz}$. 


\section{Blithfield test rig}

The Blithfield test rig is $120 \mathrm{~m}$ long and is made from high-performance polyethylene (HPPE) pipe buried at a depth of approximately $0.8 \mathrm{~m}[16,18]$. It has an outer radius and wall thickness of $80 \mathrm{~mm}$ and $9.85 \mathrm{~mm}$, respectively. The measurement positions were at access points $\mathrm{P} 1$ and $\mathrm{P} 2$ which are $30 \mathrm{~m}$ apart, with the leak located at $\mathrm{P} 1$, which was created by opening a small globe valve attached to the end of a standpipe connected to the underground hydrant valve, as shown in Fig. 2(b). Two synchronized time histories were recorded at a sampling frequency of $5 \mathrm{kHz}$ for 60 seconds.

\section{São Paulo test rig}

The São Paulo test rig is a close circuit system where a centrifugal pump is used to pressurize the system. The PVC pipe is buried at a depth of approximately $0.5 \mathrm{~m}$ with an outer radius and wall thickness of $35.8 \mathrm{~mm}$ and $3.4 \mathrm{~mm}$, respectively [8,19]. The measurement positions were at access points $\mathrm{P} 1$ and P2 with the leak located $1.25 \mathrm{~m}$ away from P1 as shown in Fig. 2(c). Two synchronized time histories were recorded at a sampling frequency of $12.8 \mathrm{kHz}$ for 60 seconds.

\section{Results}

The results are shown in the following figures:

- $\quad$ Fig. 3. Ottawa test rig using hydrophones

- $\quad$ Fig. 4. Ottawa test rig using accelerometers

- $\quad$ Fig. 5. Blithfield test rig using accelerometers

- $\quad$ Fig. 6. São Paulo test rig using accelerometers

Each figure shows the following six subplots: (a) spectrum of the two acquired signals;

(b) the coherence between the two signals; (c) magnitude of CPSD function between the 
two signals; (d) phase of CPSD function (e) magnitudes of the FRFs $H_{1}$ and $H_{2}$ using Eqs. 4(a,b); (f) spectrum of the leak noise estimated using Eqs. 1(a,b,c) highlighting the content within the frequency band of interest and depicting amplitudes at a range of $40 \mathrm{~dB}$.

Subplots (a-e) illustrate the behaviour of the signals, and the quantities that are used in the estimates of the spectra of the leak noise at source, which are given in subplots (f). Note, that also plotted on the plots of the leak spectra is a thick green dotted-dashed line representing the trend expected [14], which is proportional to $\omega^{-1}$. When processing the data, a Hanning window was used with 50\% overlap, and for convenience the frequency resolution of the spectra presented is $1 \mathrm{~Hz}$. It can be seen that a bandwidth is marked in each figure. This is the bandwidth in which the leak noise propagates directly from the source and is measured at access points $\mathrm{P} 1$ and P2. Outside this bandwidth the measured signals are dominated by other sources of noise, from the environment, for example. The bandwidth can be determined from the unwrapped phase of the CPSD. If the leak noise is detected at both sensors, and if the leak noise has propagated directly from the sources at a constant velocity, such that there are no resonance effects or wave reflections in the pipe, then the unwrapped phase between the signals changes linearly with frequency [6]. This behavior is evident in subplot (d) in Figs. 3-6. To determine when the phase deviates from a straight-line, abrupt changes in the phase are detected using the procedure detailed in $[20,21]$, and the resulting bandwidths are given in Tab. 3 .

As mentioned in Section 2, to estimate the leak noise spectrum, the FRFs $H_{1}$ and $H_{2}$ need to be estimated, which are assumed to involve only a decaying propagating wave, as can be seen by examining Eqs. (4a,b). This assumption is only valid within the bandwidth discussed above. Thus, although the processed signals are plotted over a wide- 
range of frequencies, the estimate of the leak noise shown in subplot (f) in Figs. 3-6 is only valid within the bandwidths indicated in the figures.

\section{Discussion}

The measurements from each test rig are discussed in turn, then some general comments are made about the predicted leak spectra. Note, that the effects of the soil and the pipe properties on the bandwidth over which leak noise is measured is not discussed here, as this has been treated in refs. $[8,16,19]$.

For the Ottawa test rig, measurements were conducted using both hydrophones and accelerometers and these results are shown in Figs. 3 and 4, respectively. As mentioned previously, for pressure measurements the shape and the amplitude of the leak noise spectrum can be estimated, but if accelerometers are used then only the shape of the leak noise spectrum can be estimated. In both cases this can only be done within a limited frequency range, when the data conforms to the model of the pipe system. Examining the PSD of the pressure measurements in Fig. 3(a), it can be seen that there are peaks in the spectra, which occur at approximately $56 \mathrm{~Hz}$ for the signal measured at $\mathrm{P} 1$ which is closer to the leak than $\mathrm{P} 2$, and $83 \mathrm{~Hz}$ for the signal measured at P2. These peaks have been attributed to resonances in the pipe system [18], and in the context of this paper, the peak at $56 \mathrm{~Hz}$ limits the highest frequency over which the leak noise spectrum can be estimated. It could be possible to extend this bandwidth by applying the method described in Gao et al. [22], but here the bandwidth is taken to be from 9 - $56 \mathrm{~Hz}$. Although good coherence is observed over a much wider bandwidth, as can be seen in Fig. 3(b), the FRF can only be modelled as a propagating wave from $9-56 \mathrm{~Hz}$, limiting the estimation of the leak noise to this frequency range. The modulus and phase of the CPSD are shown in Figs. 
3(c) and (d), respectively, which show that within this frequency range the behaviour is dominated by attenuating non-dispersive waves (since the phase has a linear-like behaviour). The quantities that combine to give the estimate of the leak noise spectrum are shown in Figs. 3(c, d) and (e), where the dynamic effects that limit the bandwidth can be clearly seen. Finally, the leak noise spectrum calculated using Eqs. (1a-c) is shown in Fig. 3(f). It can be seen that Eqs. (1a) and (1b) give almost identical results, which differ from the result calculated by Eq. (1c), which uses $\left|H_{2}\right|$. This involves a measurement much further from the leak (73.5 m) compared to the other measurement, which was 29.1 $\mathrm{m}$ from the leak, which means that there is much greater attenuation of the measured leak noise at high frequencies, due to the low-pass filtering effect of the pipe [16]. This attenuation would generally be the main factor that determines the upper frequency of the bandwidth in which the leak noise can be estimated, but in the specific case considered here, it is the resonance at approximately $56 \mathrm{~Hz}$ that determines the upper limit of the bandwidth. This resonance also makes it difficult to observe a general trend in the amplitude of the leak spectrum.

The results shown in Fig. 4 are for the same test rig, but for acceleration of the riser rather than the pressure in the pipe. It is evident from Figs. 4(a-d) that there are non-dispersive attenuating waves within the bandwidth $35-139 \mathrm{~Hz}$, which conforms to the model used for the FRFs. The low frequency limit of the bandwidth is higher than that for pressure sensors, due to the filtering effect of the accelerometer $[6,16]$. The high frequency limit is higher than that for pressure measurements, and this is because it is not limited by the dynamics of the system [18], but by very small leak noise signals at P2 at high frequencies compared to those at P1, which can be seen in Figs. 4(a) and 4(e). The estimated spectrum is plotted in Fig. 4(f). It can be seen that, as with the spectrum plotted in Fig. 4(f), apart 
from small frequency regions at the beginning and end of the bandwidth shown, there is some evidence that it decays with a frequency power law of $\omega^{-1}$.

Fig. 5 shows the results of the UK test rig in Blithfield in which acceleration was measured. In this case, the leak is at measurement position P1, which means that the only difference between the PSD at the measurement position and the leak noise is because of the effect of differentiation with respect to time as acceleration rather than pressure is measured.

The shape of the pressure PSD can thus be determined by dividing the acceleration PSD for P1 shown in Fig. 5(a) by $\omega^{4}$. Furthermore, as the leak is at P1 this is equivalent to applying Eq. (1b) setting $H_{1}=1$ in this case. Examining Fig. 5(a), it can be seen that up to approximately $138 \mathrm{~Hz}$ the PSD at P1 does not decay with frequency as does the PSD at P2 due to the low-pass filtering effect of the pipe. It does, however, decay above this frequency, and this is probably due to the local dynamics of the hydrant at the measurement point. The low frequency cut-off for the bandwidth over which the leak noise spectrum is estimated, is governed by the low acceleration levels due to the leak at low frequency, which means that the measurements are dominated by noise from the environment below $22 \mathrm{~Hz}$. Because the distance between P1 and P2 is much smaller than in the Ottawa test rig, the low-pass filtering effect of the pipe is less severe and so the bandwidth over which the leak noise is measured at the measurement points is larger, which is evident by examining the phase in Fig. 5(d). The predicted FRFs between the leak noise and the measurements are given in Fig. 5(e). The FRF related to P1 involves the effect of the accelerometer only, but the FRF related to P2 contains this effect and that 
due to the length of the pipe between P1 and P2. It is evident from Fig. 5(e) that up to approximately $50 \mathrm{~Hz}$, both FRFs are dominated by the sensors, but above this frequency the attenuation in the measured leak noise at point P2 can be clearly seen. The estimated leak noise spectrum is given in Fig. 5(f). As with the previous cases Eq. (1a) and either Eq. (1b) or (1c), depending on which sensors is closest to the leak give almost identical results. Note that the spectrum is decaying with a frequency power law of $\omega^{-1}$ within the frequency range marked, as with the Ottawa test rig.

The final data set is from São Paulo, and the results are shown in Fig. 6. In this test-rig, the pipe system and the pipe diameter are much smaller than the other two test-rigs and the surrounding soil is very stiff clay [19]. The measurements were made over $5.5 \mathrm{~m}$, which is a much shorter distance than in the other cases, so the bandwidth in which leak noise is found to be much higher, $273-746 \mathrm{~Hz}$. The reason why the lowest frequency is approximately $273 \mathrm{~Hz}$ is because below this frequency the measurements were dominated by noise from the pump that was used to pressurize the system. Apart from this, the general features seen in Fig. 6 are consistent with those for acceleration measurements in Figs. 4 and 5. In particular, the defining feature of the straight-line behavior for the bandwidth over which the method to predict the leak spectrum is evident in Fig. 6(d). Moreover, the influence of the accelerometer at low frequencies and the filtering properties of the pipe at higher frequencies can be seen in Fig. 6(e). Finally, it can also be observed that the spectrum of the leak noise in Fig. 6(f), broadly decays with a frequency power law of $\omega^{-1}$.

From the four cases considered above, it is not possible to state categorically that the leak noise spectra decays as $\omega^{-1}$ as predicted in [14]. Over the various bandwidths considered 
given Tab. 3, the difference in amplitudes of the leak spectrum between the high and low cut-off frequencies are respectively $-7.8 \mathrm{~dB},-6 \mathrm{~dB},-8 \mathrm{~dB}$ and $-4.3 \mathrm{~dB}$, which are relatively small, compared to the dynamic range of the measurements shown in Figs. 3-6. Given this evidence, for the purposes of modelling buried plastic water pipes for leak detection, it seems reasonable to assume that the leak noise is white. If there is a specific situation when the frequency range is very wide (for example when the ratio of the highest frequency to the lowest frequency in the range is ten), then it may be advisable to assume that the leak noise spectra decays as $\omega^{-1}$.

\section{Conclusions}

This paper has described a method to determine the shape of a leak noise spectrum at source in buried water pipes. It involves the use of measurements made at access points either side of a leak, that are typically made when using a leak noise correlator. Four measurements, from three different sites, have been presented to illustrate the approach. The method can only be used to predict the spectral shape within a frequency bandwidth where the leak noise propagates as a nondispersive wave. This bandwidth can be calculated by determining when there is a linear phase relationship with frequency (straight line behaviour) between the two measurements. It is found that the factors which limit the highest frequency of the band are either the dynamics of the pipe system or the very low level of the leak noise signal at the measurement position furthest from the leak. If pressure measurements are made, then both the level and shape of the leak noise spectrum can be estimated, but if accelerometers are used then only an estimate of the shape of the spectrum is possible. From the measurements presented, it is found that it is not possible to state categorically that the leak noise spectra decays according to a particular frequency power law. There is some evidence that it decays with a frequency 
power law of $\omega^{-1}$, but this is not definitive in all cases. For analytical and numerical models used to investigate leak detection and location in pipe systems, it is suggested that a white noise description of leak noise is adequate if the ratio of high to low frequency cut-off frequencies of the bandwidth is small $(<10)$. If the ratio of high to low frequency cut-off frequencies of the bandwidth is large $(>10)$ then it is probably better to assume that the leak noise spectrum decays with a frequency power law of $\omega^{-1}$.

\section{Acknowledgements}

The authors would like to thank the financial support provided by the São Paulo Research Foundation (FAPESP) under Grant number 2018/25360-3. The first author is grateful for the support from Coordination for the Improvement of Higher Education Personnel (CAPES) under Grant number 88887.374001/2019-00. The authors also thank the Brazilian water and waste management company (Sabesp) for funding this work and for providing one of the test rigs.

\section{References}

[1] S. El-Zahab, T. Zayed, Leak detection in water distribution networks: an introductory overview, Smart Water, vol. 4, no.5 (2019). Doi: https://doi.org/10.1186/s40713-019$\underline{0017-\mathrm{X}}$

[2] R. Puust, Z. Kapelan, D.A. Savic, T. Koppel, A Review of Methods for Leakage Management in Pipe Networks, Urban Water Journal, Vol. 7(1), (2010) 25-45. Doi: https://doi.org/10.1080/15730621003610878

[3] S.B. Grant, J.D. Saphores, D.L. Feldman, A.J. Hamilton, T.D. Fletcher, P.L.M. Cook, M. Stewardson, B.F. Sanders, L.A. Levin, R.F. Ambrose, A. Deletic, R. Brown, S.C. Jiang, D. Rosso, W.J. Cooper, I. Marusic, Taking the "Waste” out of "Wastewater" for Human Water Security and Ecosystem Sustainability, Science, vol. 337, no. (2012), pp. 681-686. Doi: https://doi.org/10.1126/science.1216852 
[4] O Globo, 5th June 2019, <https://g1.globo.com/economia/noticia/2019/06/05/oitoestados-do-pais-perdem-metade-ou-mais-da-agua-que-produzem-com-vazamentos-egatos-diz-estudo.ghtml>, accessed on 1st June 2020.

[5] O. Hunaidi, W.T. Chu, Acoustic characteristics of leak signals in water distribution pipes, Applied Acoustics, vol. 58, no. 3 (1999), pp. 235-254. Doi: https://doi.org/10.1016/S0003-682X(99)00013-4

[6] Y. Gao, M.J. Brennan, P.F. Joseph, J.M. Muggleton, O. Hunaidi, A model of the correlation of leak noise in buried plastic water pipes, Journal of Sound and Vibration, vol. 277, no.1-2 (2004), pp. 133-148. Doi: https://doi.org/10.1016/j.jsv.2003.08.045

[7] Y. Gao, Y. Liu, J.M. Muggleton, Axisymmetric fluid-dominated wave in fluid-filled plastic pipes: Loading effects of surrounding elastic medium. Applied Acoustics, vol. 116 (2017), pp. 43-49, Doi: https://doi.org/10.1016/j.apacoust.2016.09.016

[8] M.J. Brennan, M. Karimi, J.M. Muggleton, F.C.L de Almeida, F. K. de Lima, P.C. Ayala, D. Obata, A.T. Paschoalini, N. Kessissoglou, On the effects of soil properties on leak noise propagation in plastic water distribution pipes, Journal of Sound and Vibration, vol. 427 (2018), pp. 120-133. Doi: https://doi.org/10.1016/j.jsv.2018.03.027

[9] S. Yazdekhasti, K.R. Piratla, S. Atamturktur, A. Khan, Experimental evaluation of a vibration-based leak detection technique for water pipelines, Structure and Infrastructure $\begin{array}{llllll}\text { Engineering, } & \text { vol. } & 14 & \text { (2018), } & \text { pp. 46-55. Doi: }\end{array}$ https://doi.org/10.1080/15732479.2017.1327544

[10] S. El-Zahab, E.M. Abdelkader, T. Zayed, An accelerometer-based leak detection system, Mechanical Systems and Signal Processing, vol. 108 (2018), pp. 276 - 291. Doi: https://doi.org/10.1016/j.ymssp.2018.02.030

[11] J.D. Butterfield, V. Meruane, R.P. Collins, G. Meyers, S.B.M. Beck, Prediction of leak flow rate in plastic water distribution pipes using vibro-acoustic measurements, Structural Health Monitoring, vol. 17, no. 4 (2018), pp. 959-970. Doi: https://doi.org/10.1177/1475921717723881

[12] R.A. Cody, P. Dey, S. Narasimhan. Linear prediction in water distribution networks, J. Pipeline Syst. Eng. Pract., 1(1): 04019043 (2020), Doi: https://doi.org/10.1061/(ASCE)PS.1949-1204.0000415 
[13] J.D. Butterfield, A. Krynkin, R.P. Collins, S.B.M. Beck, Experimental investigation into vibro-acoustic emission signal processing techniques to quantify leak flow rate in plastic water distribution pipes, Applied Acoustics, vol. 119 (2017), pp. 146 - 155. Doi: https://doi.org/10.1016/j.apacoust.2017.01.002

[14] A.S. Papastefanou, P.F. Joseph, M. J. Brennan, Experimental investigation into the characteristics of in-pipe leak noise in plastic water filled pipes, Acta Acustica united with Acustica, vol. 98 (2012), pp. 847-856. Doi: https://doi.org/ 10.3813/AAA.918568

[15] Y. Gao, M.J. Brennan, P.F. Joseph, J.M. Muggleton, O. Hunaidi, On the selection of acoustic/vibration sensors for leak detection in plastic water pipes, Journal of Sound and Vibration, vol. 283 no. 3-5 (2005), pp. 927-941. Doi: https://doi.org/10.1016/j.jsv.2004.05.004

[16] F.C.L. Almeida, M.J. Brennan, P.F. Joseph, S. Whitfield, S. Dray, On the acoustic filtering of the pipe and sensor in a buried plastic water pipe and its effect on leak detection: An experimental investigation, Sensors, vol. 14, no. 3 (2014), pp. 5595-5610. Doi: https://doi.org/10.3390/s140305595

[17] O. Hunaidi, W.T. Chu, A. Wang and W. Guan, Detecting leaks in plastic water distribution pipes, Journal of the American Water Works Association, vol. 92, no. 2 (2000), pp. 82-94. Doi: https://doi.org/10.1002/j.1551-8833.2000.tb08819.x

[18] F.C.L. Almeida, M.J. Brennan, P.F. Joseph, Y. Gao, A.T. Paschoalini, The effects of resonances on time delay estimation for water leak detection in plastic pipes, Journal of Sound and Vibration, vol. 420 (2018), pp. 315 - 329. Doi: https://doi.org/10.1016/j.jsv.2017.06.025

[19] O. Scussel, M.J. Brennan, J.M. Muggleton, F.C.L de Almeida, A.T. Pascoalini, Estimation of the bulk and shear moduli of soil surrounding a plastic water pipe using measurements of the predominantly fluid wave in the pipe, Journal of Applied Geophysics, $\quad$ 164, (2019), pp. 237-246. Doi: https://doi.org/10.1016/j.jappgeo.2019.01.010

[20] M. Lavielle, Using penalized contrasts for the change-point problem. Signal Processing, vol. 85, no.8 (2005), pp. 1501-1510. Doi: https://doi.org/10.1016/j.sigpro.2005.01.012 
[21] R. Killick, P. Fearnhead, I.A. Eckley. Optimal detection of changepoints with a linear computational cost. Journal of the American Statistical Association, vol. 107, no. 500 (2012), pp. 1590-1598. Doi: https://doi.org/10.1080/01621459.2012.737745

[22] Y. Gao, M.J. Brennan, Y. Liu, F.C.L. de Almeida, P.F. Joseph, Improving the shape of the cross-correlation function for leak detection in a plastic water distribution pipe using acoustic signals. Applied Acoustics, vol. 127, (2017), pp.24-33. Doi: http://dx.doi.org/10.1016/j.apacoust.2017.05.033 
Figures

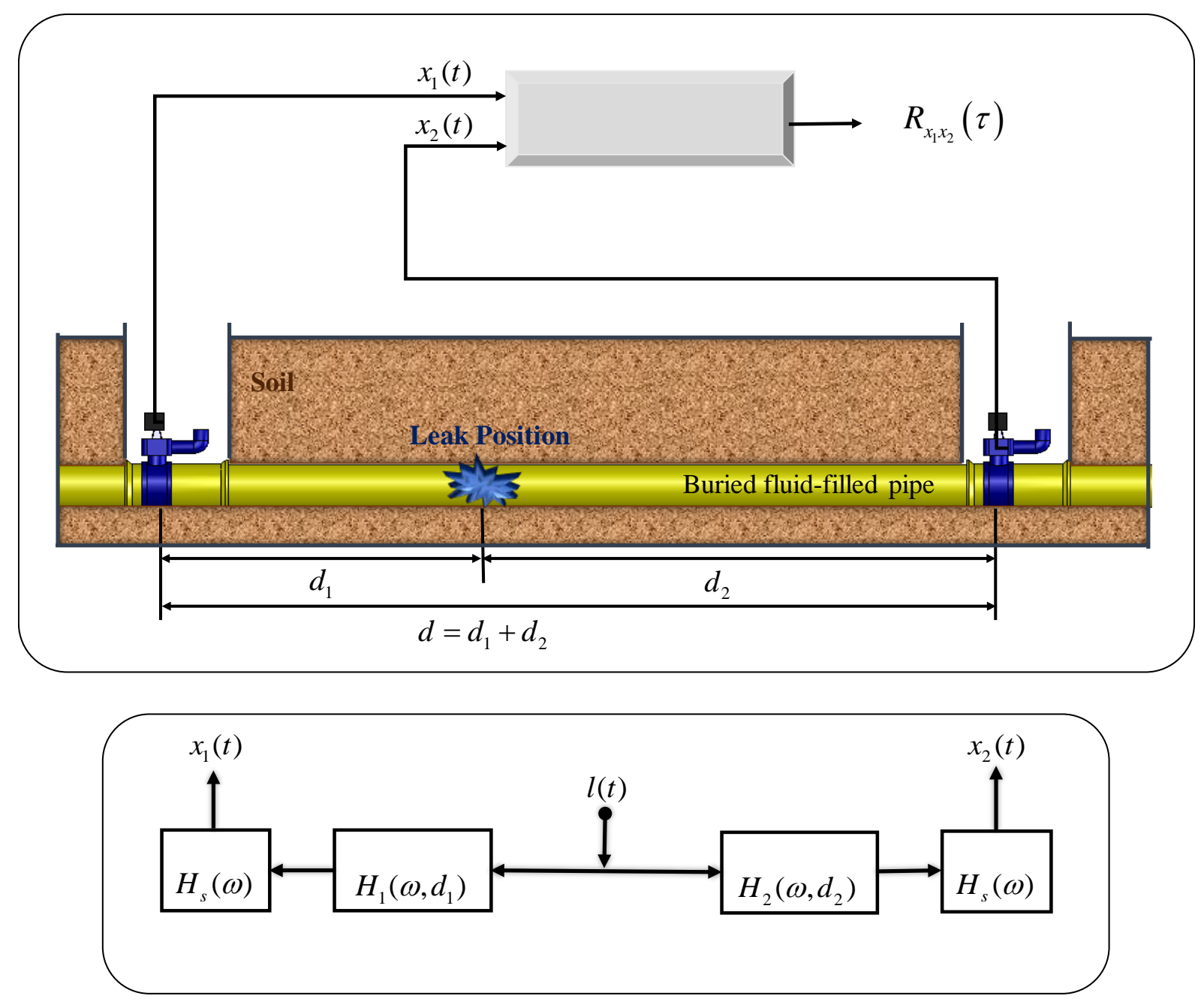

Figure 1. (a) Schematic of the process of leak detection in a buried water pipe using vibroacoustic signals for a leak bracketed by two sensors, (b) Block diagram of the pipe-system. 


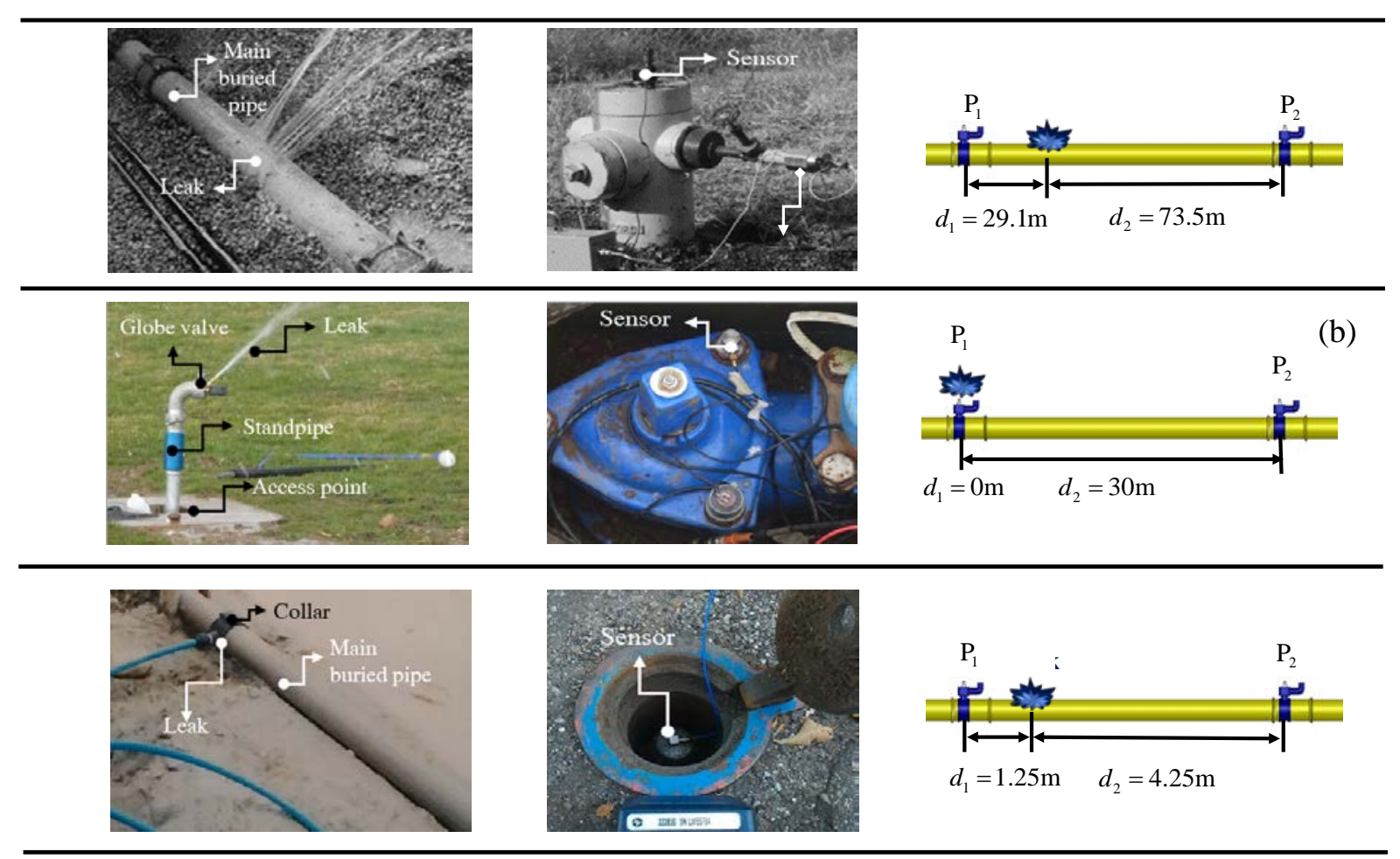

Figure 2. Schematic diagrams of the three test-rigs (not to scale) showing the source leak mechanism, the access points and corresponding distance between the sensors: (a) OttawaCanada, (b) Blithfield-UK and (c) São Paulo-Brazil. 


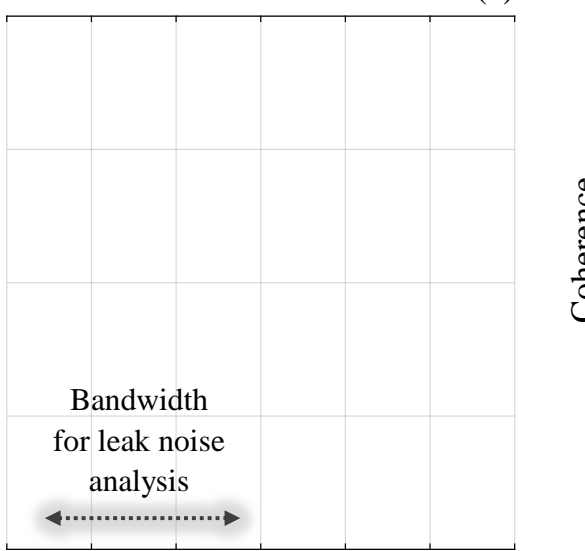

Frequency [Hz]

(c)

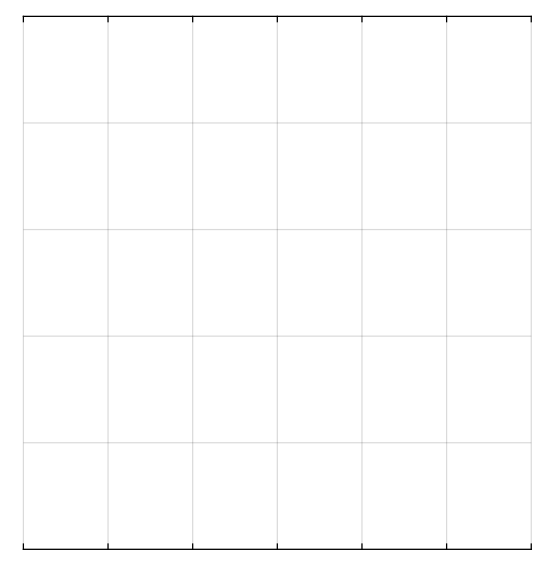

Frequency [Hz]

(e)

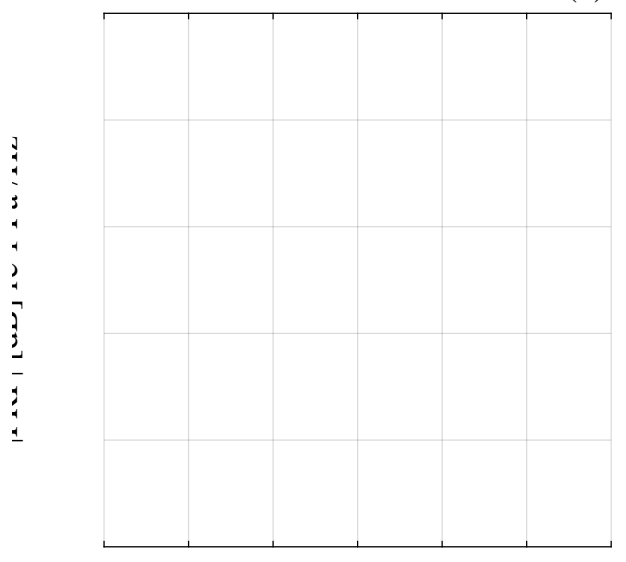

Frequency [Hz]

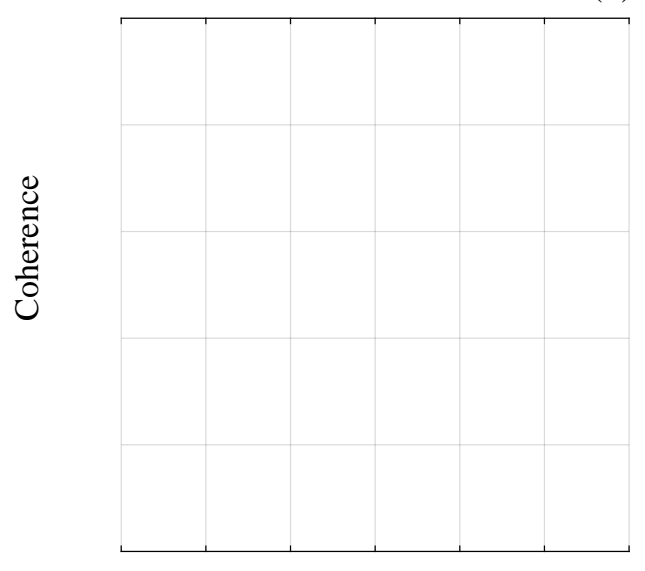

Frequency $[\mathrm{Hz}]$

(d)

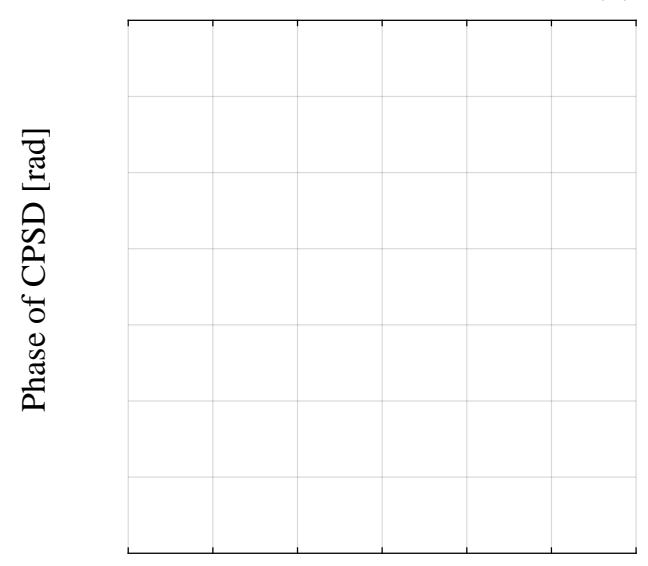

Frequency [Hz]

(f)

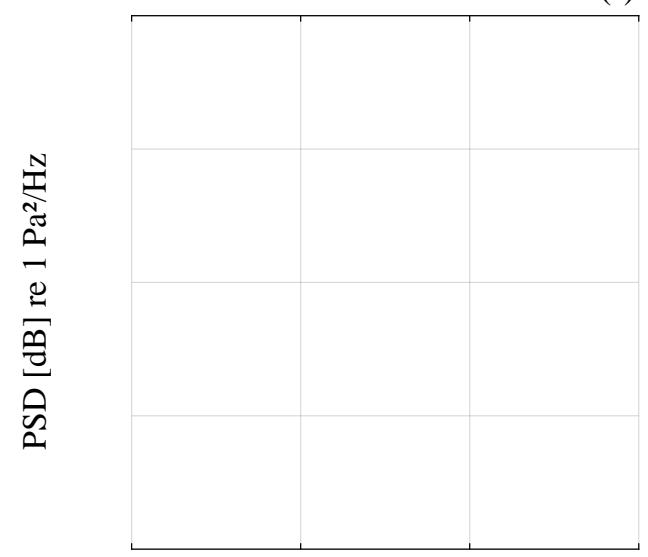

Frequency [Hz]

Figure 3. Canadian data measured with hydrophone: (a) PSD of the measured signals at P1 (black line) and P2 (blue line with circles), (b) Coherence, (c) Magnitude of CPSD, (d) Phase of CPSD, (e) Magnitude of the predicted FRF of each sensor based on Eq. (4a) (thick black line) and on Eq. (4b) (blue line with circles), (f) Predicted spectrum of the leak noise based on: Eq. (1a) (solid-thick red line), Eq. (1b) (dashed black line), Eq. (1c) (thin blue line) and the decay behaviour with a frequency power law of $\omega^{-1}$ (dashed-thick green line). Frequency bandwidth of $9-56 \mathrm{~Hz}$ (shaded grey region). 
(a)

(b)

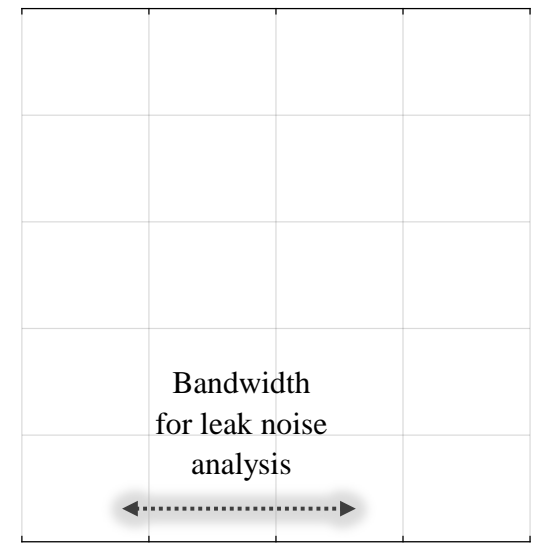

Frequency $[\mathrm{Hz}]$

(c)

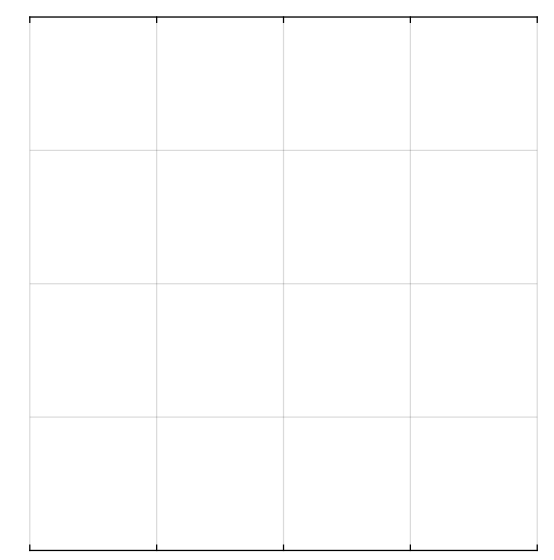

Frequency $[\mathrm{Hz}]$

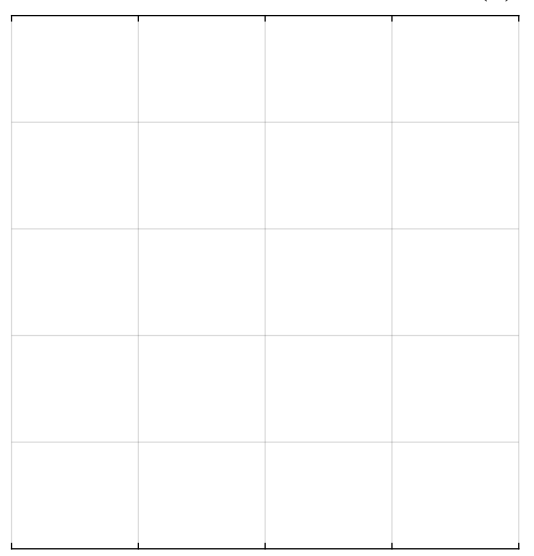

Frequency $[\mathrm{Hz}]$

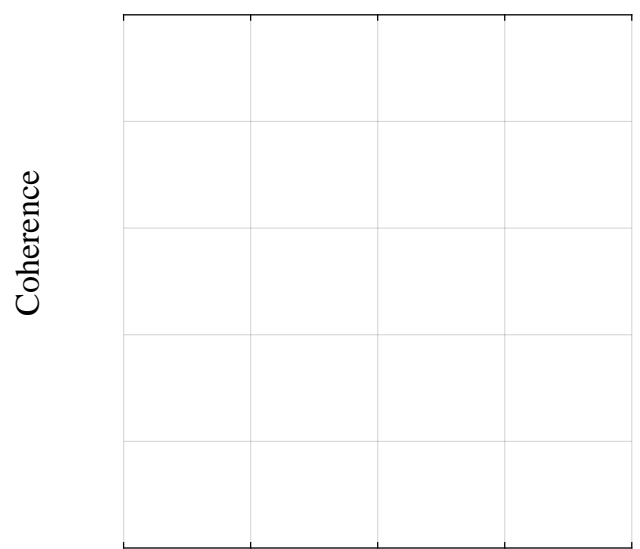

Frequency $[\mathrm{Hz}]$

(d)

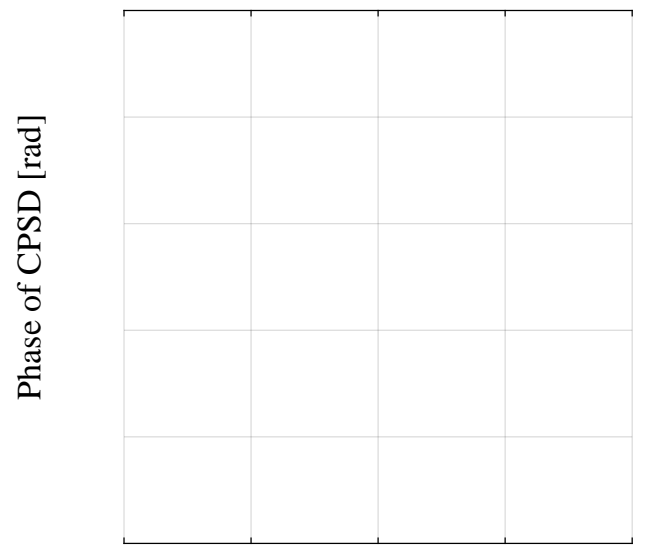

Frequency $[\mathrm{Hz}]$

(f)

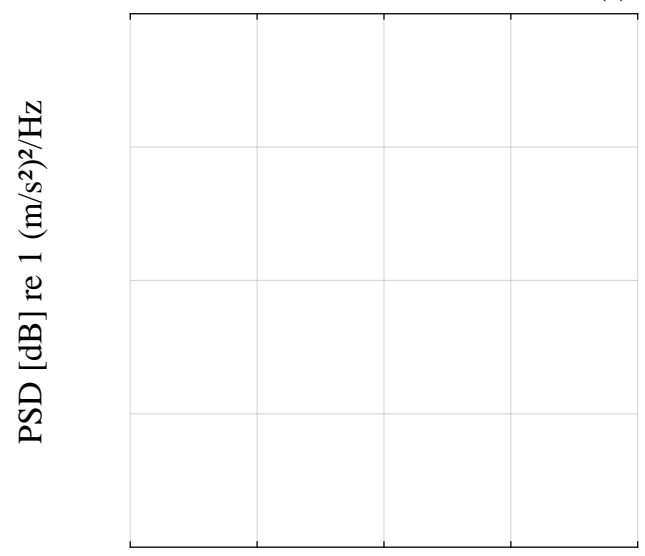

Frequency $[\mathrm{Hz}]$

Figure 4. Canadian data measured with accelerometers: (a) PSD of the measured signals at P1 (black line) and P2 (blue line with circles), (b) Coherence, (c) Magnitude of CPSD, (d) Phase of CPSD, (e) Magnitude of the predicted FRF of each sensor based on Eq. (4a) (thick black line) and on Eq. (4b) (blue line with circles), (f) Predicted spectrum of the leak noise based on: Eq. (1a) (solid-thick red line), Eq. (1b) (dashed black line), Eq. (1c) (thin blue line) and the decay behaviour with a frequency power law of $\omega^{-1}$ (dashed-thick green line). Frequency bandwidth of $35-139 \mathrm{~Hz}$ (shaded grey region). 


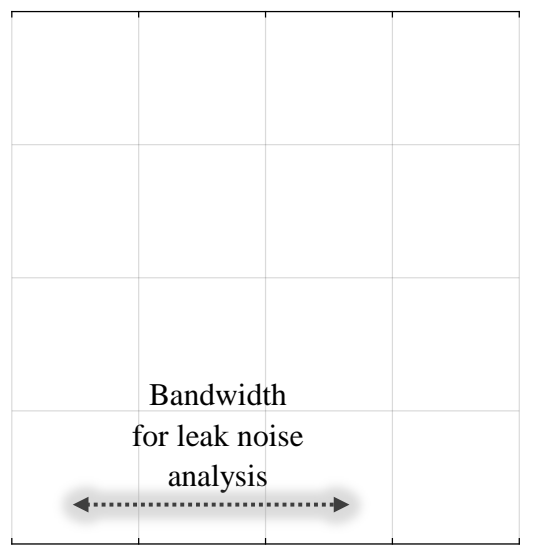

Frequency [Hz]

(c)

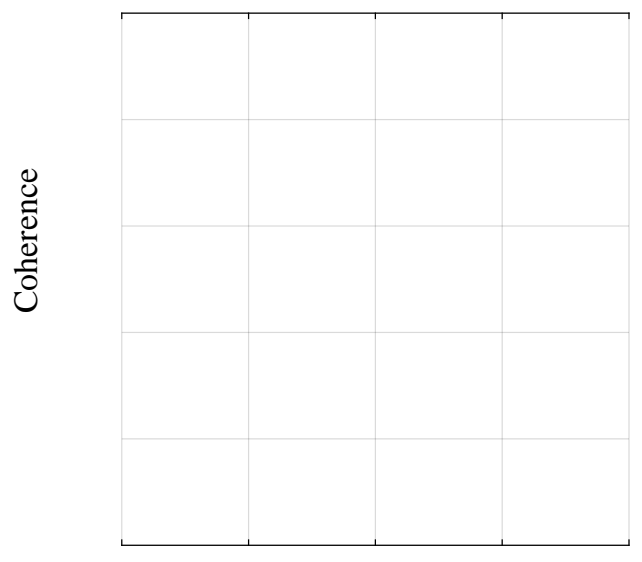

Frequency [Hz]

(d)

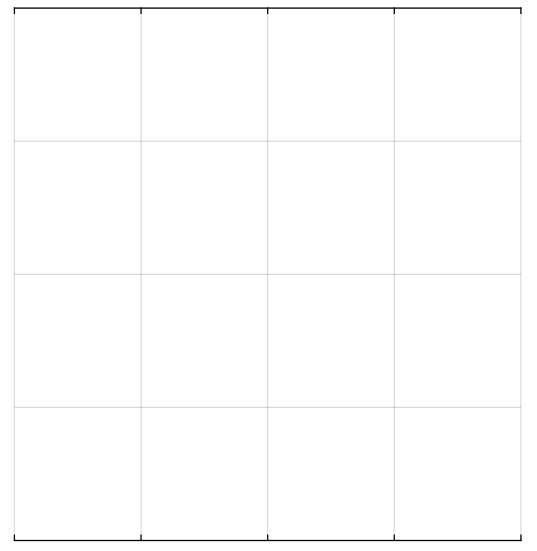

Frequency [Hz]

(e)

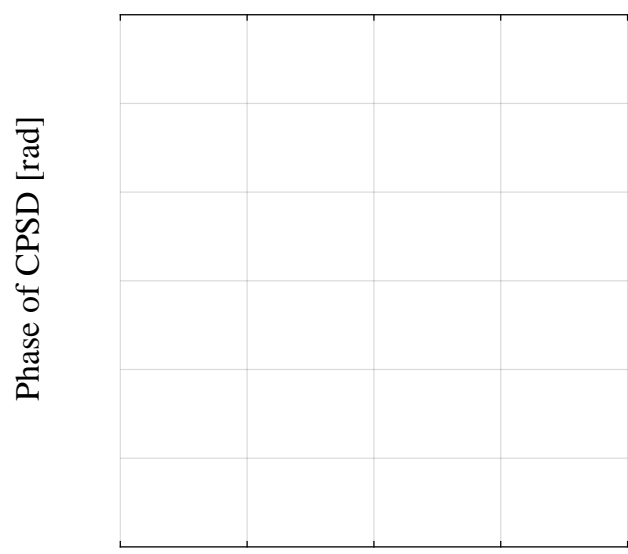

Frequency [Hz]

(f)

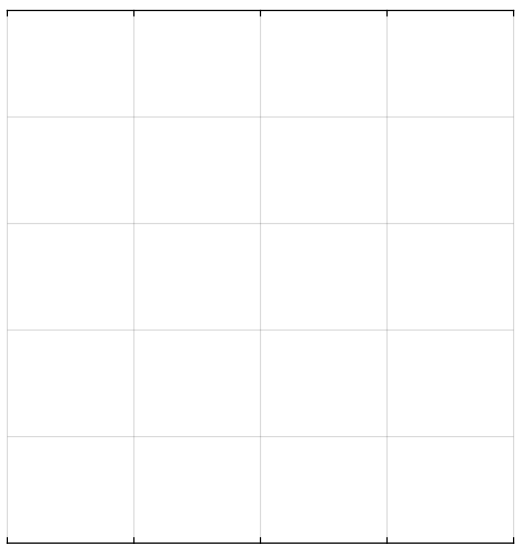

Frequency [Hz]

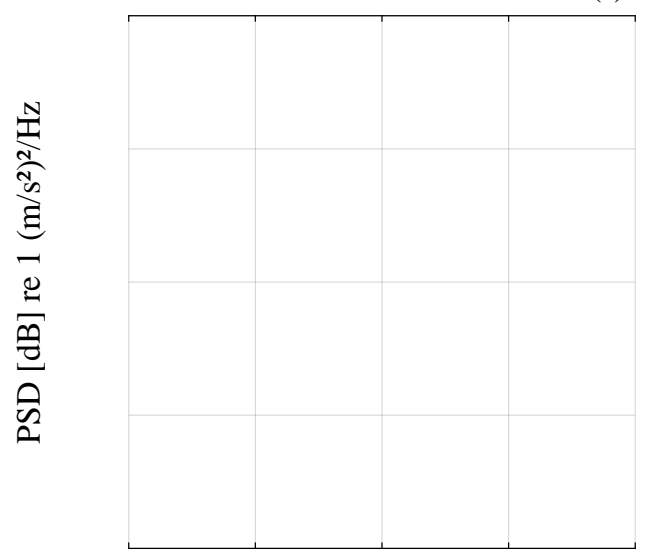

Frequency [Hz]

Figure 5. Blithfield data measured with accelerometers: (a) PSD of the measured signals at P1 (black line) and P2 (blue line with circles), (b) Coherence, (c) Magnitude of CPSD, (d) Phase of CPSD, (e) Magnitude of the predicted FRF of each sensor based on Eq. (4a) (thick black line) and on Eq. (4b) (blue line with circles), (f) Predicted spectrum of the leak noise based on: Eq. (1a) (solid-thick red line), Eq. (1b) (dashed black line), Eq. (1c) (thin blue line) and the decay behaviour with a frequency power law of $\omega^{-1}$ (dashed-thick green line). Frequency bandwidth of $22-138 \mathrm{~Hz}$ (shaded grey region). 
(a)

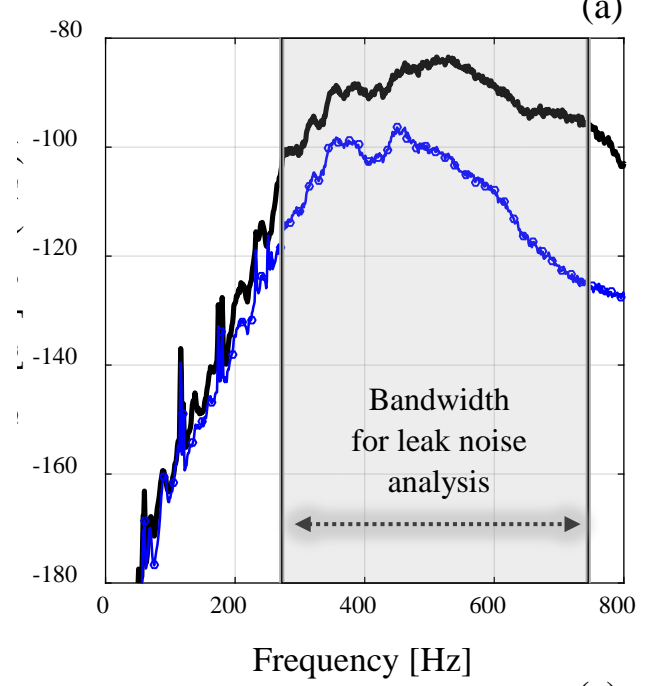

(c)
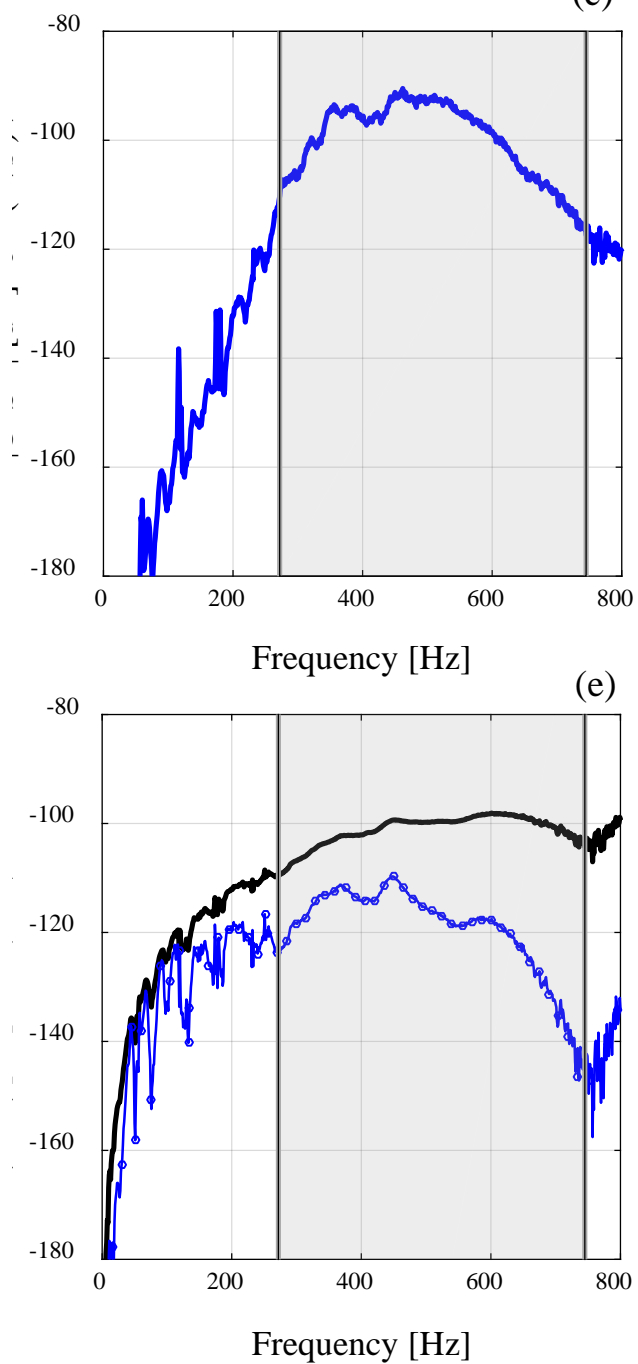

(b)

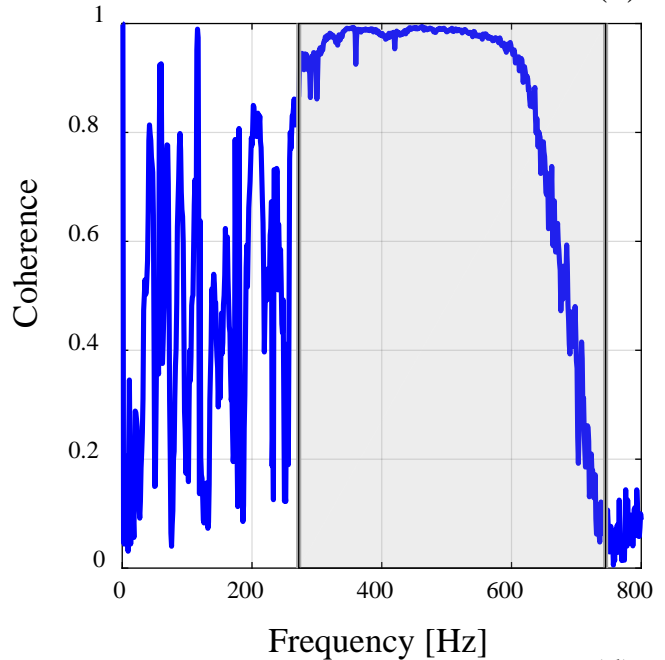

(d)

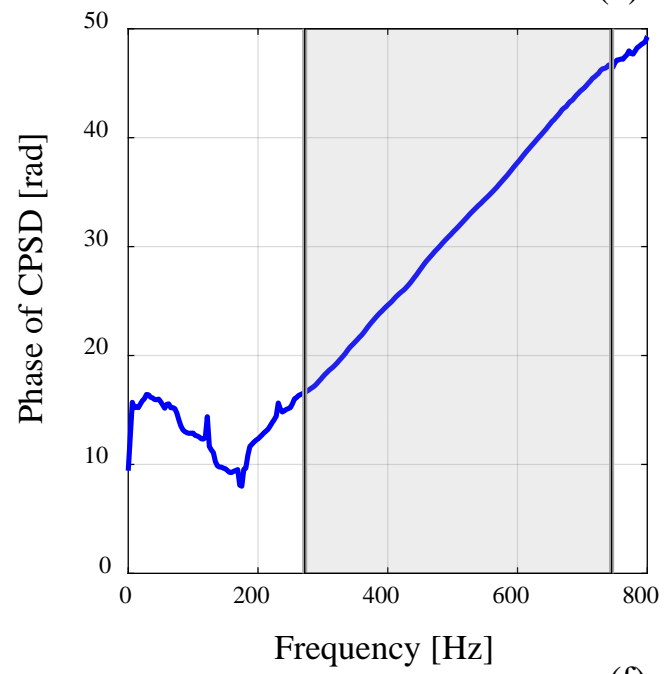

(f)

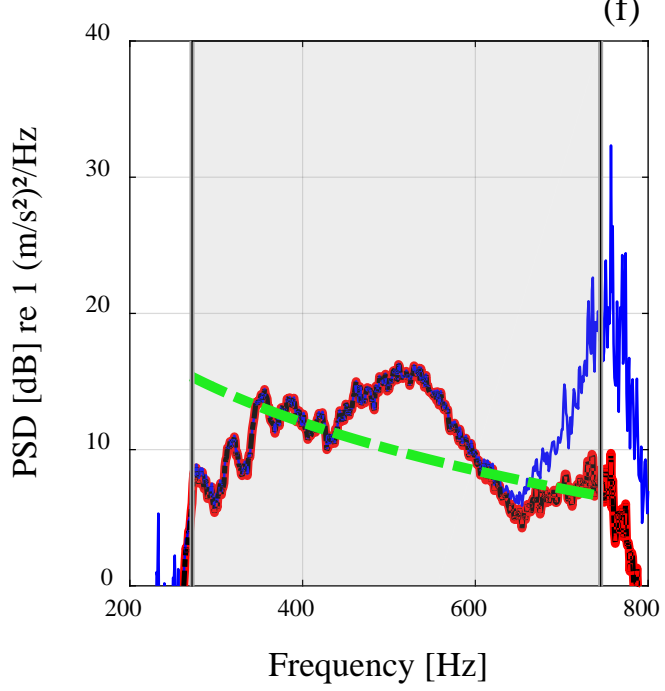

Figure 6. São Paulo data measured with accelerometers: (a) PSD of the measured signals at P1 (black line) and P2 (blue line with circles), (b) Coherence, (c) Magnitude of CPSD, (d) Phase of CPSD, (e) Magnitude of the predicted FRF of each sensor based on Eq. (4a) (thick black line) and on Eq. (4b) (blue line with circles), (f) Predicted spectrum of the leak noise based on: Eq. (1a) (solid-thick red line), Eq. (1b) (dashed black line), Eq. (1c) (thin blue line) and the decay behaviour with a frequency power law of $\omega^{-1}$ (dashed-thick green line). Frequency bandwidth of $273-746 \mathrm{~Hz}$ (shaded grey region). 
Tables

\begin{tabular}{c|c|c|c}
\hline Pipe Properties & Ottawa & Blithfield & São Paulo \\
\hline Young's modulus, $E_{\text {pipe }}\left(\mathrm{N} / \mathrm{m}^{2}\right)$ & $4.18 \times 10^{9}$ & $1.78 \times 10^{9}$ & $4.3 \times 10^{9}$ \\
Density $\rho_{\text {pipe }}\left(\mathrm{kg} / \mathrm{m}^{3}\right)$ & 900 & 900 & 900 \\
Loss factor $\eta_{\text {pipe }}$ & 0.04 & 0.06 & 0.06 \\
Poisson's ratio $v_{\text {pipe }}$ & 0.4 & 0.4 & 0.4 \\
Pipe radius $(\mathrm{mm})$ & 75 & 80 & 35.8 \\
Pipe-wall thickness $(\mathrm{mm})$ & 9.85 & 9.85 & 3.4 \\
\hline
\end{tabular}

Table 1. Pipe properties of each experimental test rig. The pipe properties for the Ottawa test rig are given in [6], the pipe properties for Blithfield test rig are given in [16] and the pipe properties for the São Paulo test rig are given in [19].

\begin{tabular}{c|c|c|c}
\hline Soil properties & Ottawa & Blithfield & São Paulo \\
\hline Bulk modulus, $B_{\text {soil }}\left(\mathrm{N} / \mathrm{m}^{2}\right)$ & $4.0 \times 10^{8}$ & $1.36 \times 10^{8}$ & $4.0 \times 10^{9}$ \\
Shear modulus, $G_{\text {soil }}\left(\mathrm{N} / \mathrm{m}^{2}\right)$ & $1.0 \times 10^{5}$ & $3.2 \times 10^{7}$ & $1.44 \times 10^{8}$ \\
Bulk and shear loss factor & 0 & 0.06 & 0 \\
Density $\rho_{\text {soil }}\left(\mathrm{kg} / \mathrm{m}^{3}\right)$ & 2000 & 2000 & 2000 \\
Poisson's ratio & 0.5 & 0.39 & 0.49 \\
\hline
\end{tabular}

Table 2. Soil properties of each experimental test rig. The soil properties for the Ottawa test rig are estimated by the authors based on unpublished analysis, the soil properties for Blithfield test rig are given in [16] and the soil properties for the São Paulo test rig are given in [19].

\begin{tabular}{l|l}
\hline $\begin{array}{l}\text { Test rig } \\
\text { (sensor) }\end{array}$ & Bandwidth \\
\hline $\begin{array}{l}\text { Ottawa } \\
\text { (pressure) }\end{array}$ & $9-56 \mathrm{~Hz}$ \\
$\begin{array}{l}\text { Ottawa } \\
\text { (acceleration) }\end{array}$ & $35-139 \mathrm{~Hz}$ \\
$\begin{array}{l}\text { Blithfield } \\
\text { (acceleration) }\end{array}$ & $22-138 \mathrm{~Hz}$ \\
$\begin{array}{l}\text { São Paulo } \\
\text { (acceleration) }\end{array}$ & $273-746 \mathrm{~Hz}$ \\
\hline
\end{tabular}

Table 3. Leak noise bandwidths. 